BMJ Open

Ophthalmology

\section{Studies on the prevalence of blindness in Ethiopia: a protocol for the systematic review and meta-analysis}

To cite: Markos M, Kefyalew B, Tesfaye HB. Studies on the prevalence of blindness in Ethiopia: a protocol for the systematic review and meta-analysis. BMJ Open Ophthalmology 2021;5:e000881. doi:10.1136/ bmjophth-2021-000881

Received 20 August 2021 Accepted 7 September 2021

A Check for updates

(C) Author(s) (or their employer(s)) 2021. Re-use permitted under CC BY-NC. No commercial re-use. See rights and permissions. Published by BMJ.

${ }^{1}$ Department of Public Health, Woldia University, Woldia, Ethiopia

${ }^{2}$ Department of Ophthalmology and Optometry, Hawassa University, Hawassa, Ethiopia ${ }^{3}$ Department of Early Childhood Care and Education, University of Gondar, Gondar, Ethiopia

Correspondence to Merkineh Markos; markosmerkineh330@gmail. com

\section{ABSTRACT}

Introduction Blindness refers to a lack of vision and/ or defined as presenting visual acuity worse than $3 / 60$ in the better eye. Its highest proportion has been conforming to the developing countries such as Ethiopia. So, timely information is crucial to design strategies. However, the study on the magnitude of blindness in Ethiopia was outdated, that means it was conducted in 2005-2006. Therefore, this protocol has been proposed to estimate the pooled prevalence of blindness in Ethiopia to provide upto-date, comprehensive evidence on this theme.

Methods and analysis The following databases will be used to search articles: PubMed, Cochrane Library, Google Scholar and retrieving references. Standard data extraction approach will be employed and presented using Preferred Reporting Items for Systematic Review and Meta-Analysis. The Newcastle-0ttawa Scale quality assessment tool will be used to evaluate the quality of studies. Analysis will be held using STATA V.11. Funnel plot and Egger's regression test will be applied to check for the potential sources of bias. Heterogeneity among the studies will be tested using Higgins method in which $\mathrm{I}^{2}$ statistics will be calculated and compared with the standard. Meta-regression and subgroup analysis will be done to identify the potential sources of heterogeneity. Cross-sectional and survey studies conducted in Ethiopia and published in English language will be included.

Ethics and dissemination Ethics approval and consent are not required. On completion, the result will be submitted to a reputable peer-reviewed journal.

Trial registration number CRD42021268448.

\section{INTRODUCTION}

Blindness refers to a lack of vision which may happen suddenly or over a period of time due to many reasons, ${ }^{1}$ while the WHO defined blindness as presenting visual acuity worse than $3 / 60$ in the better eye. ${ }^{2}$ Its causes vary from region to region and from country to country. ${ }^{3}$ The causes of blindness have been either avoidable with prevention and treatment or unavoidable, ${ }^{4}$ though the highest percentage of avoidable blindness has been corresponding to developing countries ${ }^{5}$ like Ethiopia. So, timely information is crucial to design strategies that address the life quality of sightless individuals.

\section{Rationale of the current systematic review}

The magnitude of blindness is increasing globally due to an increasing number of population and longer life expectancy. ${ }^{6}$ This statement may be true for our country Ethiopia. However, the study on the magnitude of blindness in Ethiopia was outdated (ie, done in 2005-2006), ${ }^{7}$ while different studies have been conducted at the different segments of the country. Therefore, we draft this protocol to estimate the pooled prevalence of blindness in Ethiopia to provide up-to-date, comprehensive evidence on this theme.

\section{METHODS}

Objective

To conduct a systematic review and metaanalysis on the pooled prevalence of blindness in Ethiopia.

\section{Review question}

- What is the pooled prevalence of blindness in Ethiopia?

\section{Eligibility criteria \\ Inclusion criteria}

Based on CoCoPop mnemonic (Condition, Context and Population) approach, given that articles that meet the following criteria will be included in this systematic review and meta-analysis.

1. Ethiopia inhabitants irrespective of their age, occupation, level of education and gender.

2. Papers in which blindness was measured either based on best corrected visual acuity or presenting visual acuity using any type of optotype (eg, LogMAR, Snellen E chart, etc).

3. Studies published only in English Language from 1990 to 2021.

4. This review will include all cross-sectional and survey studies that conducted using probability sampling techniques.

5. Studies only done in Ethiopia will be included. 
Exclusion criteria

1. Editorials, reports, meta-analysis, systematic reviews, case reports, case series, proceedings and grey literature.

2. Studies with participants who are not human.

\section{Databases and searching strategy}

We will search articles from the following databases which are directly related to the subject matter under review. So, PubMed, Cochrane Library, Google Scholar and retrieving references from a list of eligible studies will be employed. Additionally, we will conduct supplemental search of databases through manual searching of the reference list of all included papers and reviews. We will apply Boolean operator, that is, "AND", "NOT" and "OR". Through consideration of Boolean operator, we searched as follows ("Blindness" OR 'Blindness' [Mesh Terms] OR "Blindness" [All Fields] "Vision loss" [Mesh Terms] OR "total blindness" [Mesh Terms] OR "avoidable blindness" [All Fields] OR "functional blindness" [All Fields] OR "treatable blindness" [All Fields] OR "preventable blindness" [All Fields] OR "needless blindness"[All Fields] OR "Visual impairment" [All Fields] AND "Ethiopia" [All Fields].

\section{Data extraction and management}

Standard systematic searching will be conducted from those databases. Studies conducted before 1990, conducted in countries other than Ethiopia and unrelated articles based on their title will be excluded. Those potentially eligible studies will be imported to EndNote V.7 and exact duplicates will be removed. Two independent reviewers will do the abstract and full-text review and data abstraction. In case of disagreement between the two reviewers, discussion will be made to reach consensus. Lastly, studies will be screened and selected for full-text reviews based on the following inclusion criteria. The Preferred Reporting Items for Systematic Review and Meta-Analysis (PRISMA) statement for reporting systematic review and meta-analysis will be used to present the study inclusion, exclusion and reason for exclusion information in a diagram. The extraction will include: author, publication status, region in the country where the study was conducted, study design, year of publication, sample size and frequency of participants with blindness. Excel software will be used for data storage.

\section{Quality assessment}

To evaluate the quality of the studies, authors will use the Newcastle-Ottawa Scale quality assessment tool. ${ }^{8}$ Using the tool as a protocol, the reviewers (MM, BK and HBT) will use the blinded review approach to evaluate the quality of the original articles. Those studies with medium (fulfilling $50 \%$ of quality assessment criteria) and high quality ( $\geq 6$ out of 10 scales) will be included for analysis, while it should be noted that low-quality studies will be excluded from the meta-analysis. Conflicting ratings will be resolved by taking the mean score of the two reviewers to reach on consensus among reviewers.

\section{Statistical analysis}

The necessary data will be extracted from the studies using Microsoft Excel V.2013 format and then will be analysed by using STATA V.11 software. The primary articles will be summarised by using tables and forest plot. The researchers will calculate the SE of the prevalence of blindness for each original article using the formula $\sqrt{ }(\mathrm{P}$ $(100 \mathrm{P}) / \mathrm{N}$ ) (where, $\mathrm{P}$ is prevalence of blindness for each original article, $\mathrm{N}$ is sample size of that specific study, $\sqrt{ }=$ the square root). According to the degree of heterogeneity between the results of primary studies ( $\mathrm{I}^{2}$ index), fixed-effects or random-effects models will be applied to present the results of the pooled prevalence of blindness. If any heterogeneity is observed, it will be considered as high $(\geq 75 \%)$, moderate $(25 \%-75 \%)$ and low $(25 \%$ or less). ${ }^{9}$ The possible source(s) of heterogeneity will be investigated by using meta-regression models. Point and pooled estimates as well as their 95\% CIs will be illustrated by forest plots. Potential publication bias will be assessed subjectively by funnel plot and objectively using Egger's weighted correlation at $5 \%$ significance level. ${ }^{10}$ If the publication bias is noticed in the random-effects model, further estimation would be carried out using Duval and Tweedie's trim and fill analysis.

\section{Subgroup analysis}

Subgroup analysis will be conducted based on region, year of publication, age group, sample size and metaregression output.

\section{Presenting and reporting the results}

We will use a PRISMA flow chart to summarise the study selection process and to show the excluded and rationale for exclusion. The prevalence of blindness will be presented in a forest plot and/or individual tables.

\section{The strengths and limitation of the study}

- The drafted systematic review and meta-analysis will provide an overall prevalence of blindness in Ethiopia.

- The proposed systematic review and meta-analysis will stick to the PRISMA guidelines, ensuring the reliability in reporting and the full systematic review.

- The proposed systematic review and meta-analysis will offer the prevalence of blindness in regions of Ethiopia.

- The limitation of this study is that it does not include literature published other than in English language.

\section{CONCLUSIONS}

These systematic review and meta-analyses data will be extracted using a structured data extraction form and presented using tables. The name of authors, publication status, region, study design, year of publication, sample size and frequency of participants with blindness will be summarised. Before analysis, conversion of prevalence and its SE will be made. 
Contributors MM-conception of research protocol, study design and drafting the manuscript. BK and HBT—designing methods and analysis techniques and reviewing the manuscript. All the authors have read and approved the manuscript.

Funding The authors have not declared a specific grant for this research from any funding agency in the public, commercial or not-for-profit sectors.

Competing interests None declared.

Patient consent for publication Not applicable.

Ethics approval Ethics approval and consent are not required. On completion, the result will be submitted to a reputable peer-reviewed journal.

Provenance and peer review Not commissioned; internally peer reviewed.

Data availability statement Data are available upon reasonable request. All data relevant to the study are included in the article or uploaded as supplemental information. Not applicable.

Open access This is an open access article distributed in accordance with the Creative Commons Attribution Non Commercial (CC BY-NC 4.0) license, which permits others to distribute, remix, adapt, build upon this work non-commercially, and license their derivative works on different terms, provided the original work is properly cited, appropriate credit is given, any changes made indicated, and the use is non-commercial. See: http://creativecommons.org/licenses/by-nc/4.0/.
ORCID iD

Merkineh Markos http://orcid.org/0000-0003-1422-9837

\section{REFERENCES}

1 Medical Encyclopedia. Blindness and vision loss 2021.

2 World Health Organization. Fact sheets detail in blindness and visual impairment 2021.

3 Vaishali KV, Vijayalakshmi P. Understanding definitions of visual impairment and functional vision. Community Eye Health Journal 2021;33:S16-17.

4 Dunning $\mathrm{H}$. Leading causes of 'avoidable blindness' identified as cases set to increase. The Lancet Global Health 2017.

5 Resnikoff S, Pascolini D, Etya'ale D, et al. Global data on visual impairment in the year 2002. Bull World Health Organ 2004;82:844-51.

6 Flaxman SR, Bourne RRA, Resnikoff S, et al. Global causes of blindness and distance vision impairment 1990-2020: a systematic review and meta-analysis. Lancet Glob Health 2017;5:e1221-34.

7 Berhane Y, Worku A, Bejiga A. Prevalence and causes of blindness and low vision in Ethiopia. Ethiop J Health Dev 2007;21:204-10.

8 Newcastle-Ottawa: Scale customized for cross-sectional studies.

9 Higgins JPT, Thompson SG. Quantifying heterogeneity in a metaanalysis. Stat Med 2002;21:1539-58.

10 Sterne JA, Egger M. Funnel plots for detecting bias in meta-analysis: guidelines on choice of axis. J Clin Epidemiol 2001;54:1046-55 